\title{
Special Issue of APWeb-WAIM 2018
}

\author{
Yi Cai ${ }^{1}$ Jianliang $\mathrm{Xu}^{2} \cdot$ Qing $\mathrm{Li}^{3}$
}

Published online: 1 April 2019

(c) The Author(s) 2019

We are pleased to present a special issue of Data Science and Engineering (DSE), which contains a collection of three extended papers from the APWeb-WAIM 2018 conference. Besides these three special issue papers, this DSE issue also has three regular research papers.

APWeb-WAIM conferences focus on research, development, and applications in relation to Web information management, including a wide range of topics, such as text analysis, graph data processing, social networks, recommender systems, information retrieval, data streams, knowledge graph, data mining and application, query processing, machine learning, database and Web applications, big data, and blockchain. APWeb-WAIM 2018 was held in Macau during July 23-25, 2018, and attracted a total of 168 research paper submissions. The conference program committee selected 39 full research papers, 31 short papers, and six demonstration papers to be presented at the conference and published in the conference proceedings $[1,2]$. The conference program also included keynote presentations by Prof. Xuemin Lin (The University of New South Wales, Australia), Prof. Lei Chen (The Hong Kong University of Science and Technology, Hong Kong, SAR China), and Prof. Ninghui Li (Purdue University, USA) as well as industrial invited talks by Dr. Zhao Cao (Huawei Blockchain) and Jun Yan (YiDu Cloud).

Yi Cai

ycai@scut.edu.cn

Jianliang Xu

xuj1@Comp.HKBU.Edu.HK

Qing Li

csqli@comp.polyu.edu.hk

1 School of Software Engineering, South China University of Technology, Guangzhou, China

2 Department of Computer Science, Hong Kong Baptist University, Kowloon Tong, Hong Kong

3 Department of Computing, The Hong Kong Polytechnic University, Hung Hom, Hong Kong
The three extended papers for this special issue were selected from among all the accepted papers by the special issue guest editors Yi Cai, Jianliang Xu, and Qing Li, based on the relevance to the journal and the reviews of the conference version of the papers. The authors were asked to revise the conference paper for journal publication and in accordance with customary practice of adding $30 \%$ new materials. The revised papers again went through the review process in accordance with DSE guidelines and are finally presented to the readers in the present form.

The three extended papers in this special issue cover a variety of topics related to data science and engineering. In the first paper, "DMDP: A Dynamic Multi-source based Default Probability Prediction Framework", authors introduce an important factor to assess the credit risk of listed companies on a stock market and present a dynamic forecasting framework to predict the default probability of a company. The second paper, "Multi-Task Learning for Abstractive and Extractive Summarization", presents a general unified framework for abstractive summarization which incorporates extractive summarization as an auxiliary task. Finally, in "Efficient Subgraph Matching on Large RDF Graphs Using MapReduce", authors present an efficient distributed method to answer subgraph matching queries on big RDF graphs using MapReduce, and experiments on both synthetic and real-world datasets show that the proposed method outperforms the close competitors S2X and SHARD by an order of magnitude on average.

We hope that the readers enjoy this special issue. We would like to acknowledge the work done by all authors and their willingness to contribute their papers for this special issue. We thank all the reviewers for their expert comments and assistance in timely reviews. Finally, a note of thanks to DSE editors X. Sean Wang and Elisa Bertino for their guidance and support in this process.

Open Access This article is distributed under the terms of the Creative Commons Attribution 4.0 International License (http://creativecommons.org/licenses/by/4.0/), which permits unrestricted use, distribution, and reproduction in any medium, provided you give appropriate 
credit to the original author(s) and the source, provide a link to the Creative Commons license, and indicate if changes were made.

\section{References}

1. Cai Y, Ishikawa Y, Xu J (2018) Web and big data-second international joint conference, APWeb-WAIM 2018, Macau, China,
July 23-25, 2018, Proceedings, Part I. Lecture Notes in Computer Science 10987, Springer 2018. ISBN: 978-3-319-96889-6

2. Cai Y, Ishikawa Y, Xu J (2018) Web and big data-second international joint conference, APWeb-WAIM 2018, Macau, China, July 23-25, 2018, Proceedings, Part II. Lecture Notes in Computer Science 10988, Springer 2018. ISBN: 978-3-319-96892-6 